\title{
iBoccia: a Framework to Monitor the Boccia Gameplay in Elderly
}

\author{
Vinícius Silva, João Ramos, Filomena Soares, Paulo Novais, Pedro Arezes, Filipe \\ Sousa, Joana Silva, and António Santos
}

\begin{abstract}
The increase of the elderly population has an enormous effect on the health care system of a country, as the rise of this population sets the mood to an exponential growth in assistance and care. Indeed, the inherent costs of this populational class are higher when comparing to the younger classes. Today paradigm focuses on the reduction of these costs by promoting a healthier lifestyle on all classes of the populations. Thus, the concern of a more active lifestyle is present in the elderly population, which has proven to reduce, for example, the risk of coronary problems. The stimulus on physical activity is now higher and it is possible to get several monitoring devices to keep track on the activity that was performed. Following this trend, the present paper presents a hybrid approach that employs the use of wearable devices, the Mio Fuse band and the pandlet, and a non-wearable device, the Kinect camera, to monitor elderly people during a Boccia game scenario. Preliminary tests were performed in laboratory. The results include data collected concerning a main movement that is used during a Boccia gameplay.
\end{abstract}

Key words: Activity monitoring, Boccia, Kinect, Wearable devices.

Vinícius Silva and Filomena Soares

Algoritmi Centre, Industrial Electronics Department, University of Minho, Guimarães e-mail: a65312@alunos.uminho.pt, fsoares@dei.uminho.pt

João Ramos and Paulo Novais

Algoritmi Centre, Department of Informatics, University of Minho, Braga e-mail: jramos@di. uminho.pt, pjon@di.uminho.pt

Pedro Arezes

Algoritmi Centre, Production Systems Department, University of Minho, Guimarães e-mail: parezesedps. uminho.pt

Filipe Sousa, Joana Silva, and António Santos

Fraunhofer Portugal Research Center for Assistive Information and Communication Solutions (FhP-AICOS), Porto, Portugal. e-mail: filipe.sousa@fraunhofer.pt, joana.silva@ fraunhofer.pt, antonio.silva@fraunhofer.pt 


\section{Introduction}

Nowadays, older adults are more concerned in having an active lifestyle. However, new possibilities to motivate to exercise must be explored, due to the rise of longevity leading to a larger increase of the older population. In $2013,18 \%$ of the Europeans are aged 65 or more (more than 92 million individuals), but the European Union estimates a rise of $30 \%$ by 2060 [23]. According to the last Census, in 2011, $19 \%$ of the population in Portugal are aged 65 or more [3]. Facing these statistics, it is important to develop new possibilities to motivate and monitor the biosignals of the elderly population.

Wearable devices offer the advantage of being usable inside and outside the home, since they are small, lightweight, and less expensive. Conversely, the success of these solutions depends upon the willingness of the user to wear them. Thus, using a passive, non-invasive, and low cost solution, such as a Kinect based system provides a better approach to monitor the older adult activity, that runs silently and un-intrusively. However, the Kinect API as it is, does not have support to actively monitor certain bio signals, such as the user heart beat during an activity. Bosi et al. [6] proposed an algorithm to estimate the heart rate but the user has to be in a standing position (being in a stationary position at $2.5 \mathrm{~m}$ of the camera). Therefore, the present work presents a hybrid approach that employs the use of wearable devices, the Mio Fuse and the pandlet, and a non-wearable device, the Kinect camera, to monitor elderly people during a Boccia game scenario.

This paper is organized as follows: in Section 2 research regarding activities and devices to promote and monitor older adults is discussed, Section 3 describes the setup proposed to monitor elderly people during a Boccia game scenario. Section 4 presents some preliminary results, and finally in Section 5 the final remarks are addressed.

\section{Background}

The latest studies on health describes higher levels of obesity and inactivity in different groups of age [8], such that older adults are overweight but also in the younger ages where it has been confirmed epidemic levels of pediatric obesity [5]. Due to the increasingly aged populations this issue becomes important for the maintenance of a healthier life in older people. Thus, physical activity is important in disease prevention and in the quality of life of this group of people since an elderly person may maintain an independent life [25].

The technological developments are constant and new devices are increasingly smaller and lightweight. Considering the activity monitoring regardless the age of the user, one may find several devices with different specifications and sizes with the goal of monitoring the physical activity of the user $[10,17,26]$.

Section 2.1 describes a game that may easily be played by the elderly (taking into consideration their physical abilities and limitations) and Section 2.2 and 2.3 
presents some approaches for monitoring the physical activity of the user, regardless his/her age.

\subsection{Boccia Game}

Boccia is a game that was initially created to be played by people with cerebral palsy, being a Paralympic Game since 1984. Nowadays this modality includes athletes with different disabilities that affect their motor skills [4]. Due to its increasing popularity this game has also been adapted to children and older people. Indeed, there are national championships with adapted rules for this class of players (see [20] for the Portuguese championship). It may be played outside or indoors and the field must follows the definitions depicted in Figure 1. Just to promote the physical activity over the people in a nursing home, the rules and the size of the field may be readapted.

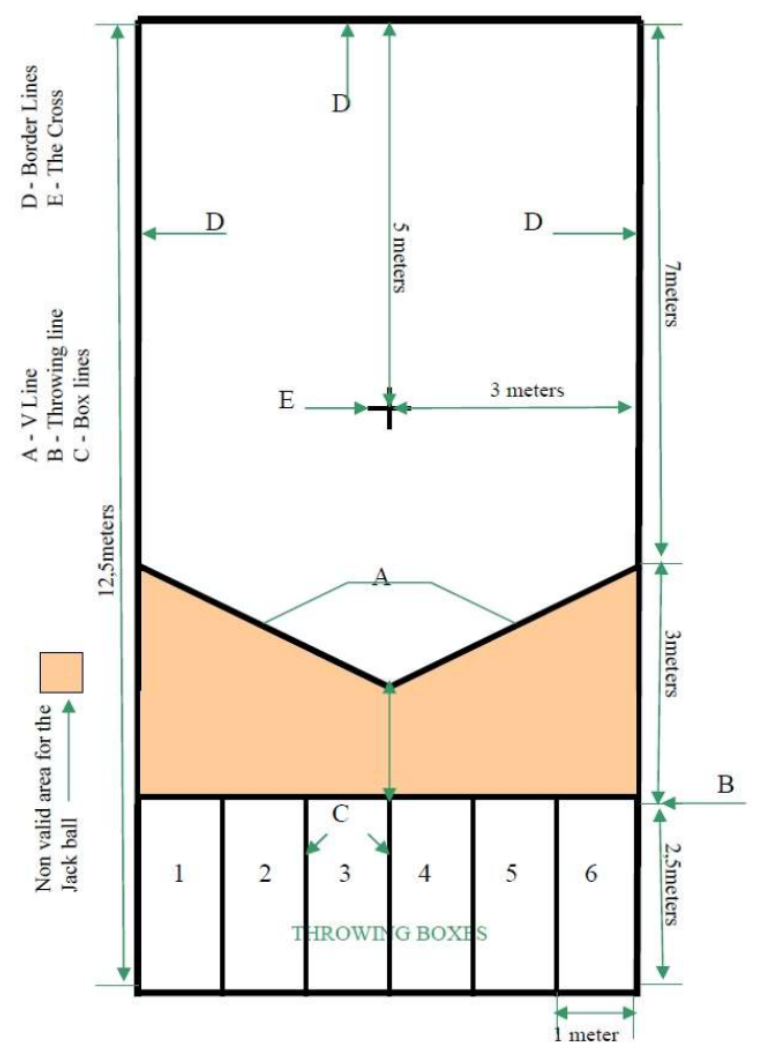

Fig. 1 Boccia field, retrieved from [21] 
Considering the goal of developing an activity and to promote the interaction between the users of a nursing home, the Boccia game is a very good option since it may be played by people with different levels of physical abilities and limitations. In each turn there are 6 players (divided in two teams) where the team with the higher scores is the winner (please refer to $[4,20]$ for details about the gameplay). In a nursing home or in its perimeter it is possible to establish a field and play this game creating different teams and promoting the interaction between the users. Thus, besides the physical activity there is also another important aspect that is the social interactions that are developed.

\subsection{Wearable Approaches}

The practice of physical activity has large benefits to the health of people. Regardless the age of the person the physical activity should be done as it may reduce disabilities (in later ages, for example), increase the independent life of the elderly and thus improve their quality of life [25].

Despite the type of exercise it is important to record the activity data so it is possible to check the performance of the user. Indeed, through the recorded data one may verify if the time of a given activity is increasing or decreasing or if the same activity may be executed for a longer time. The wearables used for this monitoring are usually equipped with accelerometers which enable the measure of the acceleration along an axis. A comprehensive review of the different types of such sensors is presented in [26]. Despite the accelerometers there are also sensors to obtain the body temperature [17], useful for example to monitoing the activity; the heart rate monitoring may be done through sound detection, just to name a few. In the wearable field there are also portable ECG (electro cardiogram) which may be used in the assessment of cardiovascular diseases [17].

Previously mentioned sensors are considered wearable, i.e., have to be with the user, which may cause some discomfort or the user may be afraid to wear it. The most common wearable devices are designed to be used in the wrist (resembling the ordinary watch $[14,10])$ being generally accepted by the user. Other wearable devices like respiratory rate monitors may be considered more invasive due to the type of sensors needed and the location where they must be on the body for the monitoring process $[12,18]$.

\subsection{Non-Wearable Approaches}

Non-wearable technologies are also available to monitor the user activity. Generally, these technologies are based on video recording systems, using one or multiple cameras to generate a 3D image $[9,27,11]$. There are different approaches employed in these systems that allows to monitor the human activity. The majority of these 
systems use classification algorithms by building a model that later allows classifying human movements [22]. A more recent approach in the literature consists in building a model of the human body and its motion via a stick-figure, i.e., a model represented by means of joints that are inter-connected with sticks $[9,27]$. However, the main drawback of this approach is the difficulty to finding extremities and joints in video sequences, since the parameter calculations requires a high computational effort [22]. A device that follows this last approach is the Microsoft Kinect sensor that along with the Microsoft Kinect Software Development Kit (SDK) computes a skeleton model of the human body in real-time [15]. Following this trend, there are several works in the literature that use Kinect for monitoring elderly people in the context of healthcare.

Dell' Acqua et. al [7] proposed the use of the Kinect camera as a basis to develop a system for monitoring physical activities in older adults. This assistive tool consists of three main components: the Kinect SDK, a Motion Analysis module, and the Activity Monitor module. Firstly, by using the first module the system extracts, in real-time, the data from the skeleton outputed by Kinect. This data is processed in the Motion Analyses module and the output data is saved in a database. Finally, the last module reads the data and performs comparisons on statistical data of the user's activities. Their approach detects if and when an older adult exergames, how often, with which intensity, and which body parts are moved most, if a person falls, and among other functionalities. A similar approach, also using Kinect for passively monitoring older adults, consisted in developing a system that performed posture recognition (siting versus standing) and gait recognition [19]. They used Support Vector Machines (SVM) as a classification method to analyze the gait and posture data obtained from Kinect. Their approach was evaluated offering promising results.

The capabilities packed in Kinect offers a compact and affordable way to use it for health monitoring and gait analysis.

\section{Proposed system}

The system proposed in this work (Figure 2) was designed to be easily expandable and start reaching other games.

\subsection{Architecture}

The software architecture is based on a three-tier framework that organizes modules (games) into dynamic categories, acting as a launcher and providing them a set of APIs for common functionalities such as sensor communication, user profile and game session management. The proposed system architecture allows the modules to be agnostic on which sensors are currently supported and being used, as well as which backend server may be used to manage user profiles and store game session 


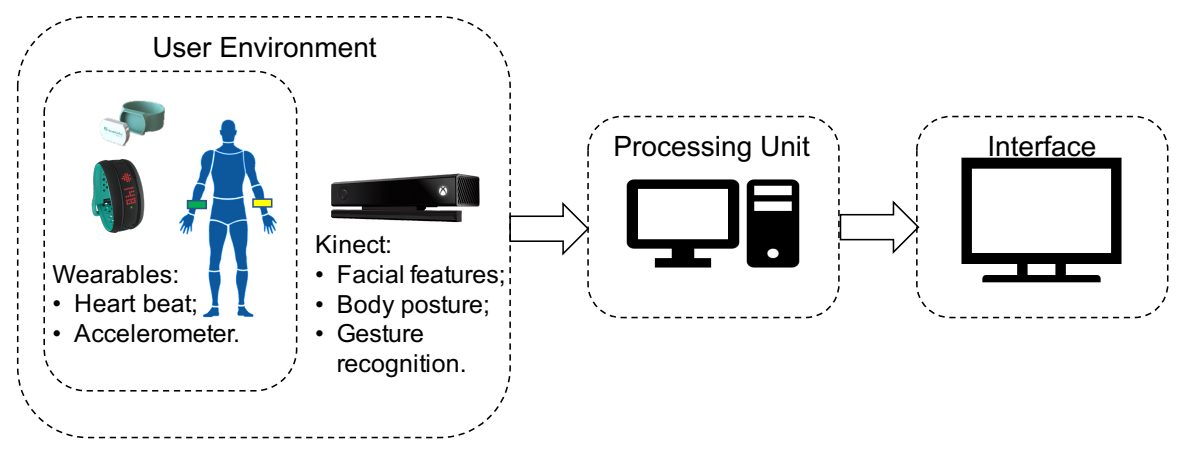

Fig. 2 System overview

data. The API [13] for sensor communication provided by the framework is implemented as shared library/DLL in pure native code $(\mathrm{C}++)$ and supports most of the existing platforms: Windows, Linux and Android. The library is responsible for all communication details with any sensor. The received data from sensors is processed and merged into a single protocol and send to any client (game) that asked for it and provided a callback. The protocol considers data from inertial sensors, gestures, body tracking, among others.

\subsection{Sensors}

The main motivation behind this project is to be used in community or nursing home unsupervised contexts, using standard and relatively inexpensive equipment to detect movements during a Boccia game scenario. Therefore, currently there is support for a small set of available sensor devices like Kinect sensor, a Mio Fuse band, pandlet [2], and a computer. The general idea lies in using the Kinect sensor, pandlet, and the Mio Fuse for actively monitoring the user in a Boccia game scenario. The Microsoft Kinect V2 is a depth sensor based on the time of light technique, that consist in measuring the time it takes for pulses of laser light to travel from a laser projector, to a target surface, and then back to the image sensor. It possesses a builtin 1080p color camera, a 3D depth sensor, and a microphone array, Figure 3. It is capable of tracking users body movement, extract facial features, and recognize gestures. The software released by Microsoft called Kinect Software Development Kit (SDK) with Application Programming Interfaces (API) gives access to raw sensor data streams as well as skeletal tracking [15]. In the present work, the face tracking, body tracking, lean tracking, and gesture recognition APIs are going to be used to track the user activity during a Boccia game scenario. The face tracking API gives access to facial features from up to six users. This facial features allows to detect if the user is happy and engaged in the activity. Additionally, it also gives accesses to facial points, necessary for example detecting the user emotional state during the 
activity. The body tracking API outputs a skeleton model composed of 25 joints. This model can be used to track each joint position that are part for example of one gesture that later can be recognized as a particular gesture in the game.

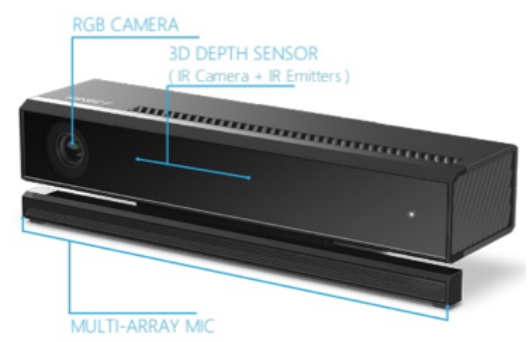

Fig. 3 The Microsoft Kinect camera, retrieved from [1]

The Pandlet (Letting Everything Sense) [2] is a bracelet with a novel architecture of embedded electronics for wireless devices that can be used to develop Wearable and IoT solutions. Pandlet includes an accelerometer, magnetometer, and gyroscope that can be used to track the user movements during the game. Along with the Kinect sensor and the pandlet, the Mio Fuse band version 2 is used in this system for monitor the user hear beat and to number of steps. It has a built-in heart rate monitor and an accelerometer [16]. Finally, combining all this information the main goal is to study the behaviour of each player during the game.

\section{Preliminary Results}

By applying the developed system to the Boccia gameplay we intend to retrieve information about the force exerted by the player during the throwing movement. This is achieved by retrieving the wrist position during different stages of the movement. Being an activity that has higher levels of acceptance by the players, which in our case are the residents of a nursing home in Guimares (Portugal), it is easier to test and access the developed system. Through it, we may measure the throwing movement regarding the acceleration of the ball, the angle that it leaves the player's hand, among others, and establish a comparison between all recorded data in order to provide the overall performance of the player. Indeed, the caregiver may verify if the user performance is increasing or not and, if the later applies, change or add a personalized treatment. Before performing the tests in the institution to validate the proposed system, a collaboration protocol between the University of Minho and the institution will be signed. Also, informed consents, describing the research purpose and methods, will be distributed and firmed by the participants or their responsible.

The Kinect sensor provides the coordinates $(x, y, z)$ for each joint of the human body. Figure 4 shows the desired angles and the selected joints that were used to 
compute them. The methodology used for computing the angles of each arm follows a previous work of the research team [24].
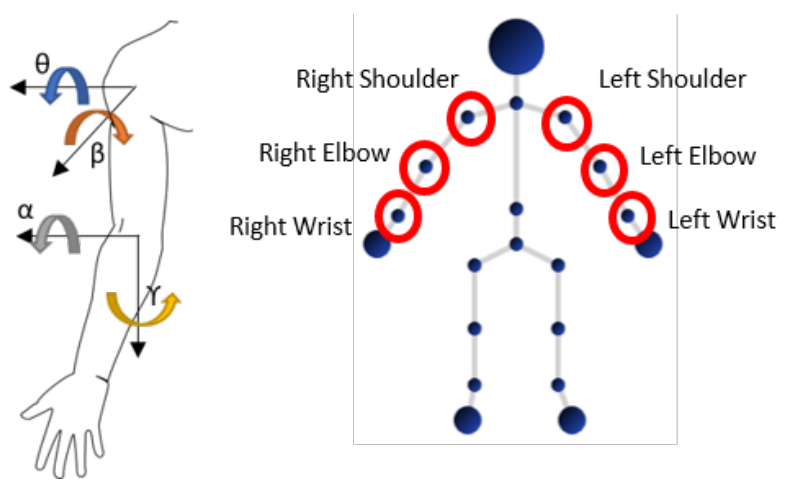

Fig. 4 On the left - The desired angles of each joint. On the right - The selected joints.

Figure 5 presents the data collected concerning a main movement that is used during a Boccia gameplay.

The performed tests were done in laboratory and the heart rate is omitted since it was constant during the throwing (due to the reduced period of time). Thus, in future tests in real game scenarious we consider to constantly monitor the heart rate of the player during the entire game play to check whether the player is in ready for his turn. Another aspect that will considered is the quality of the throwing with the

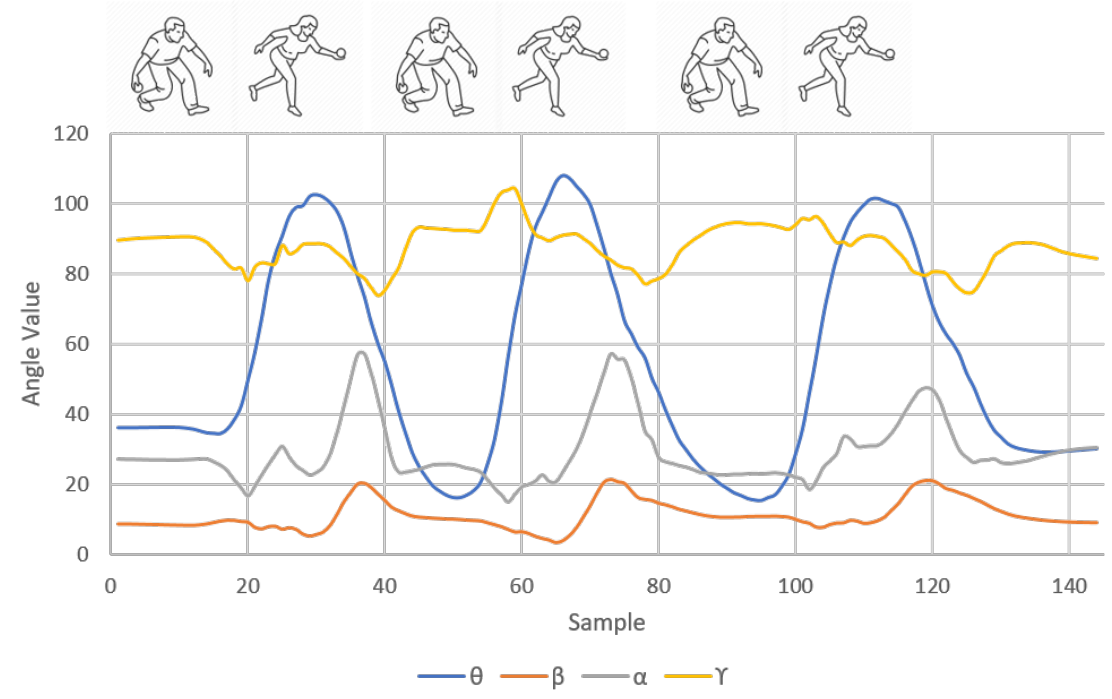

Fig. 5 Collected data of a main movement that is used during the Boccia gameplay. 
engagement of the player in the game. Indeed, when this engagement is lower, the quality of the throw usually decreases.

\section{Final Remarks}

Obesity associated to the lack of physical activity is a major concern in todays society. This problem is inherent to all groups of ages, i.e., one may find this epidemic in youth, adults and in the elderly. In this last group the concern increases since populations are getting older due to the higher number of people that are above 60 years old. A healthier life-style not only benefits the person due to the reduced risk of, for example, a coronary problem, but also the health care system of a country since the costs needed are lesser comparing to a ill person.

Besides stimulating a person to the physical activity it is also important to monitor that activity in order to check whether the person is doing the exercise in the correct way (right postures, for example) or to create activity plans to improve the quality of the exercise (do the activity in less time, for example).

We propose a hybrid system where the goal is to monitor the user activity of the elderly people while playing Boccia through a wearable device that is used on the wrist of the user and by a passive solution that monitors the environment in which the user is developing the activity.

Acknowledgements This article is a result of the project Deus ex machina: NORTE-01-0145FEDER-000026, supported by Norte Portugal Regional Operational Programme (NORTE 2020), under the PORTUGAL 2020 Partnership Agreement, through the European Regional Development Fund (ERDF).

\section{References}

1. Kinect v2 Introduction and Tutorial. https://pt.slideshare.net/ SugiuraTsukasa/kinect-v2-introduction-and-tutorial, Last accessed on Mar 13, 2017.

2. Pandlets. http://www.fraunhofer.pt/en/fraunhofer_aicos/projects/ internal_research/pandlets.html, Last accessed on Mar 22, 2017.

3. Census 2011, volume 2011. Statistics Portugal, 2011.

4. BISFed. BISFed - Boccia International Sports Federation, 2016. http://www. bi s fed. com, Last accessed on Mar 12, 2017.

5. Kristie F. Bjornson. Physical activity monitoring in children and youths. Pediatric Physical Therapy, 17(1):37-45, 2005.

6. Ilaria Bosi, Chiara Cogerino, and Marco Bazzani. Real-time monitoring of heart rate by processing of Microsoft Kinect 2.0 generated streams. In 2016 International Multidisciplinary Conference on Computer and Energy Science, SpliTech 2016, 2016.

7. Pierangelo Dell'Acqua, Leonie Verheijden Klompstra, Tiny Jaarsma, and Ali Samini. An assistive tool for monitoring physical activities in older adults. In SeGAH 2013 - IEEE 2nd International Conference on Serious Games and Applications for Health, Book of Proceedings, 2013. 
8. European Commission. European Union platform on diet, physical activity and health. Technical report, 2010. http://ec.europa.eu/health//sites/health/files/ nutrition_physical_activity/docs/eu_platform_2010frep_en.pdf, Last accessed on Jun 20, 2017.

9. Michela Goffredo, Imed Bouchrika, John N. Carter, and Mark S. Nixon. Performance analysis for automated gait extraction and recognition in multi-camera surveillance. Multimedia Tools and Applications, 50(1):75-94, 2010.

10. Fangfang Guo, Yu Li, Mohan S Kankanhalli, and Michael S Brown. An evaluation of wearable activity monitoring devices. Proc. of the 1st ACM International Workshop on Personal Data Meets Distributed Multimedia, pages 31-34, 2013.

11. A. Leu, D. Ristic-Durrant, and A. Graser. A robust markerless vision-based human gait analysis system. In SACI 2011 - 6th IEEE International Symposium on Applied Computational Intelligence and Informatics, Proceedings, pages 415-420, 2011.

12. Juan Aponte Luis, Laura M. Roa Romero, Juan Antonio Gómez-Galán, David Naranjo Hernández, Miguel Ángel Estudillo-Valderrama, Gerardo Barbarov-Rostán, and Carlos Rubia-Marcos. Design and Implementation of a Smart Sensor for Respiratory Rate Monitoring. Sensors, 2014.

13. Nuno Matos, António Santos, and Ana Vasconcelos. Kinteract: A Multi-sensor Physical Rehabilitation Solution based on Interactive Games. Proceedings of the 8th International Conference on Pervasive Computing Technologies for Healthcare, pages 350-353, 2014.

14. U. Maurer, A. Smailagic, D.P. Siewiorek, and M. Deisher. Activity recognition and monitoring using multiple sensors on different body positions. International Workshop on Wearable and Implantable Body Sensor Networks (BSN'06), pages 4-7, 2006.

15. Microsoft. Developing with Kinect, 2017. https://developer.microsoft.com/ en-us/windows/kinect/develop, Last accessed on Mar 13, 2017.

16. Mio. Mio Global: Mio Fuse Heart Rate and Sleep Monitor Activity Tracker, 2016. https://www.mioglobal.com/en-us/ Mio-FUSE-Heart-Rate-Training-Activity-Tracker/Product.aspx, Last accessed on Mar 30, 2017.

17. S C Mukhopadhyay. Wearable sensors for human activity monitoring: A review. IEEE Sensors Journal, 15(3):1321-1330, 2014.

18. Bryson Padasdao and Olga Boric-Lubecke. Respiratory rate detection using a wearable electromagnetic generator. In Proceedings of the Annual International Conference of the IEEE Engineering in Medicine and Biology Society, EMBS, 2011.

19. Monish Parajuli, Dharmendra Sharma, Dat Tran, and Wanli Ma. Senior health monitoring using Kinect. Communications and Electronics (ICCE), 2012 Fourth International Conference on, pages 309-312, 2012.

20. PCAND. Paralisia Cerebral - Associação Nacional de Desporto (PCAND), 2017. http: / / pcand. pt, Last accessed on Mar 12, 2017.

21. PCAND - Paralisia Cerebral Associação Nacional de Desporto. Regras Nacionais de Boccia Sénior 2016-2017. 2016.

22. Johannes Preis, Moritz Kessel, Martin Werner, and Claudia Linnhoff-Popien. Gait Recognition with Kinect. Workshop on Kinect in Pervasive Computing at Pervasive 2012, (July 2015):1-5, 2012.

23. Ron Davies. Older people in Europe - EU Policies and Programmes. page 8, 2014.

24. Vinícius Silva, Pedro Leite, Filomena Soares, João Sena Esteves, and Sandra Costa. Imitate me! - Preliminary tests on an upper members gestures recognition system. In Lecture Notes in Electrical Engineering, 2017.

25. Fei Sun, Ian J. Norman, and Alison E. While. Physical activity in older people: a systematic review. BMC Public Health, 13(1):449, 2013.

26. Che Chang Yang and Yeh Liang Hsu. A review of accelerometry-based wearable motion detectors for physical activity monitoring. Sensors, 10(8):7772-7788, 2010.

27. Jang Hee Yoo and Mark Nixon. Automated Markerless Analysis of Human Gait Motion for Recognition and Classification. ETRI Journal, 33(2):259-266, 2011. 